\title{
HST/WFPC2 observations of the LMC pulsar PSR B0540-69^
}

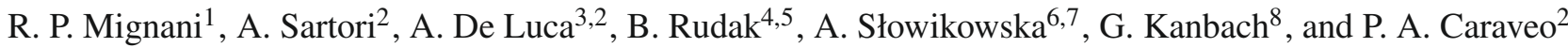 \\ 1 Mullard Space Science Laboratory, University College London, Holmbury St. Mary, Dorking, Surrey, RH5 6NT, UK \\ e-mail: rm2@mssl.ucl.ac.uk \\ 2 INAF - Istituto di Astrofisica Spaziale e Fisica Cosmica di Milano,Via Bassini 15, 20133 Milano, Italy \\ Istituto Universitario di Studi Superiori Viale Lungo Ticino Sforza 56, Pavia 27100, Italy \\ Nicolaus Copernicus Astronomical Center, ul. Rabiańska 8, 87100 Toruń, Poland \\ KAA UMK, Gagarina 11, 87-100 Torun, Poland \\ 6 Institute of Astronomy, University of Zielona Góra, Lubuska 2, 65-265 Zielona Góra, Poland \\ IESL, Foundation for Research and Technology, 71110 Heraklion, Crete, Greece \\ 8 Max-Planck Institut für Extraterrestrische Physik, Giessenbachstrasse 1, 85741 Garching bei München, Germany
}

Received 14 December 2009 / Accepted 2 March 2010

\section{ABSTRACT}

\begin{abstract}
Context. The study of the younger, and brighter, pulsars is important for understanding the optical emission properties of isolated neutron stars through observations which, even in the $10 \mathrm{~m}$-class telescope era, are much more challenging for older and fainter objects. PSR B0540-69, the second brightest $(V \sim 22)$ optical pulsar, is obviously a primary target for these investigations.

Aims. The aims of this work are several: (i) constraining the pulsar proper motion and its velocity on the plane of the sky and improving the determination of the pulsar coordinates through optical astrometry; (ii) obtaining a more precise characterisation of the pulsar optical spectral energy distribution (SED) through a consistent set of multi-band, high-resolution, imaging photometry observations and studying the relation with the X-ray spectrum, including the presence of a spectral turnover between the two bands. Last, we aim at (iii) measuring the pulsar optical phase-averaged linear polarisation, for which only a preliminary and uncertain measurement has been obtained so far from ground-based observations, and at testing the predictions of different neutron star magnetosphere models. Methods. We performed high-resolution observations of PSR B0540-69 with the Wide Field and Planetary Camera 2 (WFPC2) aboard the Hubble Space Telescope (HST), in both direct imaging and polarimetry modes.

Results. From multi-epoch astrometry we set a $3 \sigma$ upper limit of 1 mas $^{-1} r^{-1}$ on the pulsar proper motion, implying a transverse velocity $<250 \mathrm{~km} \mathrm{~s}^{-1}$ at the $50 \mathrm{kpc}$ LMC distance. Moreover, we determined the pulsar absolute position with an unprecedented accuracy of 70 mas. From multi-band photometry we characterised the pulsar power-law spectrum and derived the most accurate measurement of the spectral index $\left(\alpha_{\mathrm{O}}=0.70 \pm 0.07\right)$, which indicates a spectral turnover between the optical and X-ray bands. Finally, from polarimetry we obtained a new measurement of the pulsar phase-averaged polarisation degree $(\mathrm{PD}=16 \% \pm 4 \%)$, consistent with magnetosphere models, depending on the actual intrinsic polarisation degree and depolarisation factor, and we found that the polarisation vector $\left(22^{\circ} \pm 12^{\circ}\right.$ position angle $)$ is possibly aligned with the semi-major axis of the pulsar-wind nebula and with the apparent proper motion direction of its bright emission knot.

Conclusions. Deeper studies with the HST can only be possible with the refurbished Advanced Camera for Surveys (ACS) and with the new Wide Field Camera 3 (WFC3).
\end{abstract}

Key words. pulsars: general - pulsars: individual: PSR B0540-69

\section{Introduction}

About 40 years after identification of the optical counterpart of the Crab pulsar, only a few rotation-powered pulsars have been identified in the optical domain (see Mignani 2009a,b, for recent reviews). Of these, only four have spin-down ages of $\$ 10000$ years: the Crab pulsar, PSR B1509-58, PSR B0540-69 in the large magellanic cloud (LMC), and the Vela pulsar. The pulsar PSR B0540-69 in the large magellanic cloud (LMC) is often referred to as the Crab pulsar "twin" because it is very similar in age ( $\sim 1700$ years), spin period $(P=50 \mathrm{~ms})$, and rotational energy loss $\left(\dot{E} \sim 10^{38} \mathrm{erg} \mathrm{s}^{-1}\right)$. PSR B0540-69 is the second pulsar discovered in X-rays by the Einstein Observatory (Seward et al. 1984) and the first extragalactic pulsar detected at any wavelength. Like the Crab, PSR B0540-69 is also embedded

* Based on observations with the NASA/ESA Hubble Space Telescope, obtained at the Space Telescope Science Institute, which is operated by AURA, Inc. under contract No. NAS 5-26555. in a bright pulsar wind nebula (PWN) visible at wavelengths from the optical to the soft/hard X-rays (e.g. De Luca et al. 2007; Petre et al. 2007; Słowikowska et al. 2007). After its discovery, PSR B0540-69 has been observed by nearly all X-ray satellites, EXOSAT (Ögelman \& Hasinger 1990), Ginga (Nagase et al. 1990), ROSAT (Finley et al. 1993), BeppoSax (Mineo et al. 1999), Chandra (Kaaret et al. 2001), Rossi-XTE (de Plaa et al. 2003), ASCA (Hirayama et al. 2002), Integral (Słowikowska et al. 2007), and Swift (Campana et al. 2008). Its X-ray light curve is characterised by a single, broad peak, very much at variance with, e.g., that of the Crab pulsar. In radio, the distance to the LMC made PSR B0540-69 undetectable for a long time until pulsations were finally detected from Parkes (Manchester et al. 1993). Giant radio pulses were discovered by Johnston \& Romani (2003), aligned in phase with the peak of the X-ray pulse (Johnston et al. 2004), making PSR B0540-69 the second youngest radio pulsar to feature this phenomenon. PSR B0540-69 is also one of a handful of rotation-powered 
pulsars with a measured braking index, obtained from X-ray observations (e.g., Zhang et al. 2001; Cusumano et al. 2003; Livingstone et al. 2005).

In the optical, PSR B0540-69 is the second brightest isolated neutron star $(V \sim 22)$ identified so far. Optical pulsations were detected by Middleditch \& Pennypacker (1985) soon after the X-ray discovery, making PSR B0540-69 the third optical pulsar after the Crab (Cocke et al. 1969) and Vela pulsars (Wallace et al. 1977). However, it was only through high-resolution imaging observations with the ESO New Technology Telescope (NTT) that its optical counterpart was actually identified (Caraveo et al. 1992; Shearer et al. 1994). The PSR B0540-69 optical light curve (Middleditch et al. 1987; Gouiffes et al. 1992; Boyd et al. 1995; Gradari et al. 2009) is characterised by a single broad peak, very similar to the X-ray and radio light curves, with a significant dip on top. The optical spectral energy distribution (SED) of PSR B0540-69 was first measured by Middleditch et al. (1987) from high-speed multi-band photometry that suggested a possible excess in the $U$ band with respect to an otherwise monotonic power-law continuum $\left(F_{v} \propto v^{-\alpha_{\mathrm{O}}}\right)$.

A re-analysis of the same multi-band photometry measurements, however, did not yield any evidence of the claimed $U$-band excess (Nasuti et al. 1997). The power-law spectrum demonstrates that the optical emission from PSR B0540-69 has a magnetospheric origin, with an optical luminosity consistent with the expectations of the Pacini \& Salvati (1987) model. Low-resolution spectra of PSR B0540-69 were obtained by Hill et al. (1997) with the Hubble Space Telescope (HST) and by Serafimovich et al. (2004) with the Very Large Telescope (VLT), although the latter was affected by contamination from the supernova remnant. By using multi-band imaging photometry of PSR B0540-69 from archival HST observations, Serafimovich et al. (2004) confirmed that the optical spectrum is dominated by a power-law continuum, although with a different spectral index from the values previously published. They also report a tentative proper motion measurement for PSR B0540-69, which, however, was not confirmed by De Luca et al. (2007) using a long time baseline of HST observations. Phase-resolved polarimetry observations of PSR B0540-69 were performed by Middleditch et al. (1987) but only yielded an upper limit on the phase-averaged polarisation degree, while image polarimetry observations of Chanan \& Helfand (1990) focused on the PWN. More recently, image polarimetry observations of PSR B0540-69 were performed by Wagner \& Seifert (2000) using the VLT. They report a phase-averaged polarisation degree of $\approx 5 \%$ (with no associated error) which was probably affected by the contribution of the unresolved PWN. Thus, only preliminary, or uncertain, phase-averaged optical polarisation measurements exist for PSR B0540-69.

In this paper, we report the results of new astrometry, photometry, and polarimetry measurements of PSR B0540-69 performed with the $H S T$ as a part of a dedicated programme aimed at comprehensive study of the pulsar and of its PWN (Mignani et al., in preparation). From the pulsar astrometry, we constrain its proper motion and its velocity on the plane of the sky and provide an updated reference position for future observations. From the pulsar photometry we measure the optical SED anew and study the relation with the X-ray spectrum and verify the presence of a spectral turnover between the two energy bands. From the pulsar polarimetry, we measure its phase-averaged polarisation and test the predictions of different neutron star magnetosphere models. The paper is divided as follows. Observations and data analysis are described in Sect. 2, while the results are presented and discussed in Sects. 3 and 4, respectively.
Table 1. Summary of the HST/WFPC2 observations PSR B0540-69, with their pivot wavelength $\lambda$ and bandwidth $\Delta \lambda$ in $\AA$, and the total integration time $T$ in seconds.

\begin{tabular}{llcr}
\hline \hline $\begin{array}{l}\text { Date } \\
\text { yyyy-mm-dd }\end{array}$ & Filter & $\begin{array}{c}\lambda(\Delta \lambda) \\
(\AA)\end{array}$ & $\begin{array}{r}T \\
(\mathrm{~s})\end{array}$ \\
\hline $2007-06-21$ & $F 336 W$ & $3359(204)$ & 780 \\
& $F 450 W$ & $4557(404)$ & 780 \\
& $F 555 W$ & $5442(522)$ & 300 \\
& $F 675 W$ & $6717(368)$ & 420 \\
& $F 814 W$ & $7995(646)$ & 600 \\
& $F 606 W / P O L Q$ & $6038(651)$ & 1800 \\
$2007-07-26$ & $F 606 W / P O L Q$ & $\ldots$ & 1800 \\
$2007-09-25$ & $F 606 W / P O L Q$ & $\ldots$ & 1800 \\
$2007-11-05$ & $F 606 W / P O L Q$ & $\ldots$ & 1800 \\
$2005-11-15$ & $F 555 W$ & $\ldots$ & 480 \\
& $F 547 M$ & $5483(205)$ & 1040 \\
$1999-10-17$ & $F 336 W^{*}$ & $\ldots$ & 600 \\
& $F 791 W^{*}$ & $7872(519)$ & 400 \\
& $F 547 M^{*}$ & $\ldots$ & 800 \\
& $F 502 N$ & $5012(27)$ & 11000 \\
& $F 673 N$ & $6732(47)$ & 8200 \\
$1995-10-19$ & $F 555 W^{*}$ & $\ldots$ & 600 \\
& $F 656 N$ & $6564(22)$ & 3400 \\
& $F 658 N^{*}$ & $6591(29)$ & 4000 \\
\hline
\end{tabular}

Notes. ${ }^{(*)}$ Observations used by Serafimovich et al. (2004).

\section{Observations and data analysis}

We observed PSR B0540-69 with the Wide Field and Planetary Camera 2 (WFPC2) aboard HST (Programme \#10900, PI Mignani). The WFPC2 is a four-chip CCD detector, sensitive to radiation in the 1150-11000 $\AA$ spectral range. To exploit the maximum spatial resolution, the pulsar was centred on the Planetary Camera $(P C)$ chip which has a pixel scale of $0{ }^{\prime} 045$ and a field-of-view of $35^{\prime \prime} \times 35^{\prime \prime}$. The WFPC2 observations were performed on June 21, July 26, September 25, and November 5, 2007 for a total of six spacecraft orbits, corresponding to four different visits. Since our target is in the continuous viewing zone (CVZ), a declination zone that can be visible for an entire spacecraft orbit for a few days of the year, our programme could have been carried out in only one visit. However, we decided to split it into different visits since the narrow CVZ time window would have made it more difficult to cope with the spacecraft orientation constraints required for the WFPC2 polarimetry observations (see below).

As part of our programme, we performed both multi-band photometry and polarimetry observations (see Table 1 , top section, for a summary). To maximise the spectral coverage, we observed through the $F 336 W, F 450 W, F 555 W, F 675 W$, and $F 814 W$ filters. Observations were carried out in the first visit for two consecutive orbits of the spacecraft. The last orbit of the first visit was devoted to the first of the four planned polarimetry observations, one at each of the selected polarimetry angles. Indeed, the only possibility of performing polarimetry observations with the WFPC2 and to keep the target positioned in the same chip is to rotate the spacecraft to orient the instrument polariser. This requires that the observations must be scheduled 
in different visits for each polarimetry angle. This means that the choice of the spacecraft roll angle is limited by the guide star/solar panel constraints. Polarimetry observations were performed at nominal position angles of $0^{\circ}, 45^{\circ}, 90^{\circ}$, and $135^{\circ}$ using the $F 606 \mathrm{~W}$ filter in combination with the POLariser Quad (POLQ) filter. For each filter, all observations were split into three shorter exposures to filter out cosmic ray hits.

Additional WFPC2 observations of PSR B0540-69, taken through broad-band filters, are available in the HST archive (see Table 1, lower sections). These include two sets of $F 555 \mathrm{~W}$ observations taken on October 191995 (\#6120, PI: Caraveo) and November 15, 2005 (\#10601, PI: Lundqvist), respectively, and one set of $F 336 W$ and $F 791 W$ observations, taken on October 171999 (\#7340, PI: Morse). Observations taken through the medium-band filter $F 547 \mathrm{M}$ are available from programmes \#7340 and \#10601. Finally, observations taken through the narrow-band filters F656N, F658N, F502N, and F673N are available from programmes \#6120 and \#7340. Part of the 1995-1999 WFPC2 observations were used by Serafimovich et al. (2004) to study the pulsar optical SED. To complete the available spectral coverage, we decided to add the archival observations taken through the $F 791 W$ filter to our data set and, for comparison, those taken through the $F 336 \mathrm{~W}$ and $F 555 \mathrm{~W}$ ones. At variance with Serafimovich et al. (2004), we decided not to use the observations taken through narrow-band filters, where the pulsar is fainter and where it is more difficult to isolate its emission from that of the nebula.

All data were processed on-the-fly at the Space Telescope Science Institute (STScI) through the WFPC2 CALWP2 reduction pipeline for bias, dark, flat-field correction, flux, and polarimetry calibration using the closest-in-time reference calibration frames. For each filter, we combined single exposures with the STSDAS task combine to produce co-added and cosmic-ray free images.

\section{Results}

\subsection{Astrometry}

Firstly, we used the available high-resolution WFPC2 observations of PSR B0540-69 to obtain a compelling constraint on the pulsar proper motion through optical astrometry. To do this, we used the long time baseline provided by the observations of De Luca et al. (2007). In particular, we used all data collected with the $F 555 W$ and $F 547 M$ filters (see Table 1 ) and only considered the $P C$ images to maximise the spatial resolution. The selected data set includes five observations spanning $\sim 11.7$ years. To measure the PSR B0540-69 proper motion through relative astrometry, we applied the algorithm that we have successfully applied in a number of previous investigations including, e.g., our first study of the pulsar proper motion (De Luca et al. 2007). We started by selecting a grid of 60 good reference sources that were detected in the $P C$ field-of-view and not extended, unsaturated, with a high signal-to-noise ratio and not too close to the CCD edges. For each source, we computed their pixel coordinates on each image by fitting a 2-D Gaussian to their brightness profile, with an uncertainty of 0.02-0.06 pixels per coordinate. We evaluated the position of the pulsar optical counterpart in the same way, with an uncertainty of 0.03-0.04 pixels per coordinate. We corrected the source coordinates for the WFPC2 geometric distortion (Anderson \& King 2003), as well as for the "34th row defect" (Anderson \& King 1999). We assumed the 1995 image as a reference and aligned the associated reference grid along right ascension and declination using the known telescope roll angles. By fitting the reference star positions, we computed the coordinate transformation, which yields the best superposition of each frame grid on the 1995 one. In the fitting procedure we discarded ten reference objects yielding large residuals using an iterative sigma clipping algorithm. The resulting rms uncertainty on the superposition of the frame grids turned out to be $0.06-0.08$ pixel per coordinate (using the remaining 50 reference stars). This coordinate transformation allowed us to convert the pulsar positions to a common reference frame and to evaluate its displacement in right ascension and declination with respect to its 1995 position. A simple linear fit to the measured displacements as a function of time yields no evidence of any significant proper motion of the pulsar, with

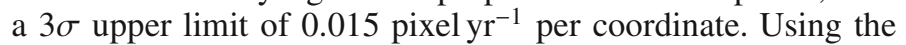
well-calibrated WFPC2 plate scale, we set a $3 \sigma$ upper limit to

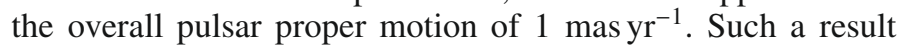
confirms our previous findings (De Luca et al. 2007), but it is slightly more compelling because of the longer time baseline (11.7 years with respect to $\sim 10$ years) covered by this data set.

Secondly, we used our new WFPC2 images to compute updated coordinates for PSR B0540-69 through optical astrometry. To this aim, we re-calibrated the image astrometry using as a reference the position of stars selected from the Two Micron All Sky Survey (2MASS) catalogue (Skrutskie et al. 2006) which has a mean positional error $\leq 0.2$ and is linked to the International Celestial Reference Frame (ICRF) with $\sim 0$ '.015 accuracy (Skrutskie et al. 2006). As discussed in Mignani et al. (2005), 2MASS is preferred to the Guide Star Catalogue 2.3 (GSC-2.3; Lasket et al. 2008) and to the US National Observatory B1.0 catalogue (USNO-B1.0; Monet et al. 2003) for fields in the LMC. On the other hand, both the USNO CCD Astrograph Catalogue 2 (UCAC-2; Zacharias et al. 2004) and its update UCAC-3 (Zacharias et al. 2009) only provide a sparse astrometric grid in the mosaic WFPC2 field-of-view. As a reference for our astrometry re-calibration, we chose the 2007 June image taken through the $F 555 \mathrm{~W}$ filter, where PSR B0540-69 is detected with a good signal-to-noise, and for which the correction for the WFPC2 geometric distortion is well modelled. We used the mosaic of the four WFPC2 chips $\left(160^{\prime \prime} \times 160^{\prime \prime}\right.$ field-of-view; 0.' 1 pixel scale $)$ since it provides a large enough field-of-view to include a sufficient number of $2 M A S S$ stars to be used for the astrometry re-calibration. We produced the mosaic image with the STSDAS task wmosaic, which also applies the correction for the geometric distortions of the four chips. Approximately $602 M A S S$ objects are identified in the mosaic WFPC2 image. As in Sect. 3.1, we filtered out extended objects, stars that are either saturated or too faint to be used as reliable astrometric calibrators, or too close to the CCD edges. We finally performed our astrometric calibration using 40 suitable $2 M A S S$ reference stars, evenly distributed in the WFPC2 field-of-view. Then, for both the $2 M A S S$ stars and the pulsar, we determined their pixel coordinates in the WFPC2 reference frame from the centroid of their intensity profile, computed by fitting a 2-D Gaussian. This yielded errors (per coordinate) of $\$ 0.01$ and of $\$ 0.05$ WFPC2 pixels on the centroids of the $2 M A S S$ stars and of the pulsar, respectively. We then computed the fit to the pixel-to-sky coordinate transformation for the $2 M A S S$ stars using the code ASTROM $^{1}$, based on higher order polynomials. By applying the computed fit to the pixel coordinates of PSR B0540-69, we finally obtained $\alpha_{\mathrm{J} 2000}=05^{\mathrm{h}} 40^{\mathrm{m}} 11.204^{\mathrm{s}}, \delta_{\mathrm{J} 2000}=-69^{\circ} 19^{\prime} 54^{\prime \prime} .34$, with an overall positional accuracy $\delta r=0^{\prime} .^{\prime} 12(1 \sigma)$. We estimated the

\footnotetext{
${ }^{1}$ http://star-www.rl.ac.uk/Software/software.htm
} 
accuracy on the computed coordinates by adding in quadrature the rms of the astrometric fit, $\sigma r \sim 0$ ! 1 , the uncertainty in the registration of the WFPC2 image on the $2 M A S S$ reference frame, $\sigma_{\text {tr }}=0{ }^{\prime} 055^{2}$, the $\sim 0{ }^{\prime} 015$ accuracy of the link of the $2 M A S S$ coordinates to the ICRF, and the uncertainty on the pulsar centroid $\left(\$ 0{ }^{\prime} 005\right)$. Since the uncertainty on the reference star centroids is much smaller than that of the pulsar, we neglected it in our global error budget.

As a check of our absolute astrometry, we used each of the F555W data sets (see Table 1), which were obtained from observations performed during different visits, i.e. with different telescope pointings and spacecraft roll angles, to obtain independent measurements of the pulsar position. Since the pivot wavelength of the $F 547 M$ filter is essentially the same as that of the $F 555 \mathrm{~W}$ one (see Table 1), the wavelength dependence of the geometric distortion should not induce any bias when using the correction optimised for the $F 555 W$ filter (see also De Luca et al. 2007). Thus, we could also use the two F547M data sets as a reference for our absolute astrometry. In particular, for the 2005 November observations, we decided to use the F547M data set instead of the $F 555 \mathrm{~W}$ one, because of its longer integration time. The other suitable medium/broad-band filter data sets cannot be formally used for an independent check of our astrometry since they were obtained from observations performed during the same visits as the $F 555 \mathrm{~W}$ and $F 547 M$ ones, with the same telescope pointing and roll angles. In principle, the data sets obtained from the observations performed through the F606W/POLQ filter, which were taken on different visits, could be used. However, possible effects of the polarisation optics on the WFPC2 astrometry, as well as the mapping of the geometric distortion, are still to be studied, thus making the use of imaging polarimetry data less suitable for astrometry (see also Kaplan et al. 2008). By re-calibrating the astrometry of each data set through the same procedure described above we obtained: $\alpha_{\mathrm{J} 2000}=05^{\mathrm{h}} 40^{\mathrm{m}} 11.208^{\mathrm{s}}$ and $\delta_{\mathrm{J} 2000}=-69^{\circ} 19^{\prime} 54^{\prime \prime} .12$ $(\delta r=0.14 ; 1 \sigma)$ for the 1995 October $F 555 W$ data set. Similarly, we obtained: $\alpha_{\mathrm{J} 2000}=05^{\mathrm{h}} 40^{\mathrm{m}} 11.193^{\mathrm{s}}, \delta_{\mathrm{J} 2000}=-69^{\circ} 19^{\prime} 54^{\prime \prime} 12$ $\left(\delta r=0 !^{\prime} 15 ; 1 \sigma\right)$ and $\alpha_{\mathrm{J} 2000}=05^{\mathrm{h}} 40^{\mathrm{m}} 11.206^{\mathrm{s}}, \delta_{\mathrm{J} 2000}=$ $-69^{\circ} 19^{\prime} 54^{\prime \prime} 10(\delta r=0.13 ; 1 \sigma)$ for the 2005 November and for the 1999 October $547 \mathrm{M}$ data sets, respectively. All four sets of coordinates are consistent within $\approx 1 \sigma$, which confirms that our first measurement was free of systematics. The marginal, although not significant, differences in the pulsar coordinates are most likely due to the different number of $2 M A S S$ stars used to compute the astrometric solution and to their relative distribution in the WFPC2 field-of-view, which depends on the different telescope pointing directions and roll angles. Marginal differences can also come from time-dependent shifts of the WFPC2 chips in the HST focal plane, which can affect astrometry of the WFPC2 mosaics. The upper limit that we obtained on the PSR B0540-69 proper motion (see Sect. 3.1) is much smaller than the error on our absolute astrometry. Thus, we can neglect any displacement of the pulsar in the 11.7 year time span covered by the available WFPC2 observations and compute an even more precise position from the average of the four sets of coordinates independently computed from the $F 555 W / F 547 M$ data sets. This yields $\alpha_{\mathrm{J} 2000}=05^{\mathrm{h}} 40^{\mathrm{m}} 11.202^{\mathrm{s}}, \delta_{\mathrm{J} 2000}=-69^{\circ} 19^{\prime} 54^{\prime \prime} .17\left(\delta r=00^{\prime} 07\right.$; $1 \sigma)$ as the most accurate value of the PSR B0540-69 position.

\footnotetext{
${ }^{2}$ As in Lattanzi et al. (1997), we defined $\sigma_{\text {tr }}=\sqrt{3} \times \sigma_{\mathrm{s}} / \sqrt{N}_{\mathrm{s}}$, where $\sqrt{3}$ accounts for the free parameters in the astrometric fit (x-scale, yscale, and rotation angle) $N_{\mathrm{s}}$ is the number of $2 M A S S$ stars and $\sigma_{\mathrm{s}}=0.2$ is the conservative mean positional error of their coordinates (Skrutskie et al. 2006).
}

\subsection{Multi-band photometry}

We used our WFPC2 observations of PSR B0540-69 to perform multi-band photometry using, for the first time, a set of observations taken consistently at the same epoch (see Table 1). This is important for minimising systematic effects on the pulsar photometry caused by possible long-term variations in the instrument zero points, to a degrade in the instrument efficiency, or to the use of different calibration data sets. In the case of PSR B0540-69, high-resolution imaging photometry observations are, at present, more appropriate than spectroscopy since they are less affected by the contamination from the bright supernova remnant (see also discussion in Serafimovich et al. 2004).

We measured the flux of PSR B0540-69 in our WFPC2 images through customised aperture photometry using the IRAF package digiphot. To maximise the signal-to-noise ratio, we measured the pulsar counts through small apertures ( 3 pixel radius) and subtracted the PWN background measured in an annulus of 10 pixel radius at a 2 pixel distance from the photometry aperture not to include the wings of the point spread function (PSF). We then applied the aperture correction using the specific coefficients given in Holtzman et al. (1995) per each filter. Observations at shorter wavelengths with the WFPC2 are affected by contaminants that progressively build up on the CCD surface (Bagget et al. 2002). The CCD contamination results in temporal variations in throughput that cause a decrease in the measured source flux. This is an issue for the measurement of the pulsar flux in the $F 336 \mathrm{~W}$ filter, where the contamination rate can be $\approx 0.06 \%$ per day and tends to be higher at the centre of each $P C$ chip, where the pulsar is positioned. This effect is not accounted for by the CALWP2 pipeline and has to be corrected manually. We then applied the contamination correction to our measurement, computed during monthly de-contamination procedures of the CCD, the last one carried out about three weeks before our observations ${ }^{3}$. To all our flux measurements we then applied correction to compensate for the time and positiondependent charge transfer efficiency (CTE) losses of the WFPC2 detector, using the formulae given in Dolphin (2009).

Following the WFPC2 Data Handbook (Bagget et al. 2002), we computed the count-rate to flux conversion using the image keywords PHOTFLAM and PHOTZPT, respectively, derived by the WFPC2 photometry calibration pipeline. We then derived magnitudes $m_{F 336 \mathrm{~W}}=21.77 \pm 0.17, m_{F 450 \mathrm{~W}}=22.09 \pm 0.05, m_{F 555 \mathrm{~W}}=$ $22.07 \pm 0.05, m_{F 675 W}=22.18 \pm 0.04$, and $m_{F 814 W}=22.35 \pm 0.03$. To complement our broad-band imaging photometry measurements, we also computed the pulsar flux from the 1999 October F791W observation (see Table 1). Using the same approach as described above, we derived $m_{F 791 W}=22.29 \pm 0.04$, which is in quite good agreement with what is obtained through the slightly redder $F 814 \mathrm{~W}$ filter. The computed magnitudes and their errors are summarised in Table 2 . The quoted formal errors are purely statistical and do not account for systematic errors related to the uncertainty of the WFPC2 photometry calibration, which are of the same order of magnitude for all filters.

To have an independent cross-check of our measurements, we also measured the pulsar flux from the archival 1999 October F336W observation and from the 2005 November and 1995 October F555W observations. Not to introduce systematic effects into our photometry, for both filters we used the same apertures and background areas used for the 2007 June observations. For the $F 336 W$ filter, we obtained $m_{F 336 W}=21.96 \pm 0.19$, i.e. very well consistent with our measurement from the 2007

\footnotetext{
${ }^{3}$ http://www.stsci.edu/hst/wfpc2/analysis
} 
Table 2. Observed WFPC2 magnitudes of PSR B0540-69 and associated errors (in parenthesis).

\begin{tabular}{|c|c|c|c|}
\hline \multirow[t]{2}{*}{ Filter } & \multirow{2}{*}{$\begin{array}{l}\text { Date } \\
\text { yyyy-mm-dd }\end{array}$} & \multicolumn{2}{|c|}{ Magnitudes } \\
\hline & & This work & $\begin{array}{l}\text { Serafimovich } \\
\text { et al. }(2004)^{*}\end{array}$ \\
\hline \multirow[t]{2}{*}{$F 336 W$} & $2007-06-21$ & $21.77(0.17)$ & \\
\hline & $1999-10-17$ & $21.96(0.19)$ & $22.25[21.98](0.13)$ \\
\hline F450W & 2007-06-21 & $22.09(0.05)$ & \\
\hline \multirow[t]{3}{*}{$F 555 W$} & 2007-06-21 & $22.07(0.05)$ & \\
\hline & 2005-11-15 & $22.11(0.04)$ & \\
\hline & 1995-10-19 & $21.99(0.03)$ & 22.17 [22.14] \\
\hline F675W & 2007-06-21 & $22.18(0.04)$ & \\
\hline$F 719 W$ & 1999-10-17 & $22.29(0.04)$ & $22.44[22.37](0.12)$ \\
\hline$F 814 W$ & $2007-06-21$ & $22.35(0.03)$ & \\
\hline
\end{tabular}

Notes. ${ }^{(*)}$ Values accounting for the CTE correction are given in brackets.

June observation. For the the $F 555 W$ filter, however, we obtained $m_{F 555 W}=22.11 \pm 0.04$ and $m_{F 555 W}=21.99 \pm 0.03$ for the 2005 November and for the 1995 October observations, respectively. Thus, while the 2007 June and 2005 November F555W measurements are in almost perfect agreement with each other, the 1995 October one shows a somewhat larger difference, between $\sim-0.08$ and $\sim-0.12 \mathrm{mag}$. Although it is not formally significant, this difference is greater than the nominal uncertainty of $0.02-0.04$ mag on the WFPC2 zero points (Heyer et al. 2004), and it implies an actual difference in the measured CTE-corrected count rate, possibly related to the on-the-fly recalibration of the WFPC2 1995 October F555W data set. We also compared our measurements with the pulsar fluxes measured by Serafimovich et al. (2004) on the very same data sets (fourth column in Table 2), i.e., for the 1995 October F555W and for the 1999 October F336W and F791W observations. Our measurements yield fluxes brighter by $\approx 0.1-0.2 \mathrm{mag}$ with respect to those of Serafimovich et al. (2004), although still compatible within the errors. We attribute this difference to their apparently not applying the CTE correction to their measurements, which can be of this order of magnitude. Indeed, by applying such correction we found that their measurements turn out to be more consistent with ours (see Table 2, values in brackets). In particular, their CTE-corrected 1995 October F555W flux measurement agrees with our 2007 June/ 2005 November F555W ones, thus confirming that the slightly deviant measurement that we obtained from the former data set might actually reflect a difference in the on-the-fly re-calibration.

\subsection{Polarimetry}

We measured the pulsar polarisation using the web interface ${ }^{4}$ to the WFPC2 polarisation calibration tool (Biretta \& Mc Master 1997). This program computes the transmission of a polariser element, for a given filter and CCD gain, using a synthetic spectrum simulated by the STSDAS package synphot. As a reference for the pulsar, we assumed its power-law spectrum determined in Sect. 3.2. Then, the program computes the Stokes $I, Q$, and $U$ parameters from the counts measured in the three images oriented along the three different polarisation angles and applies the coordinate transformation to convert the measured polarisation

\footnotetext{
4 www.stsci.edu/hst/wfpc2/software/wfpc2_pol_calib. html
}

from the detector to the sky reference frame using the telescope roll angle information.

We selected the triplet of images oriented along the three position angles of $0^{\circ}, 45^{\circ}$, and $90^{\circ}$. As in Sect. 3.2, for each image we measured the pulsar counts with the IRAF digiphot package using an aperture of 3 pixel radius and subtracted the background sampled in an annulus of 10 pixel width. Since the WFPC2 calibration tool returns the Stokes parameters as a linear combination of the counts measured in the three images (Biretta \& Mc Master 1997), this procedures accounts for the subtraction of the PWN contribution (Chanan \& Helfand 1990) to the measured pulsar polarisation. We then applied the aperture correction from Holtzman et al. (1995) and the CTE correction from Dolphin (2009) to the background-subtracted source counts. In this way, we measured the Stokes parameters of the pulsar $(Q / I)_{\mathrm{PSR}}=0.09 \% \pm 0.03 \%$ and $(U / I)_{\mathrm{PSR}}=$ $0.12 \% \pm 0.02 \%$. These correspond to a phase-averaged polarisation degree $\mathrm{PD}=15.2 \% \pm 2.5 \%$ with the polarisation vector oriented along a positional angle PA $=27.7^{\circ} \pm 10^{\circ}$ (east from north), where PA and PD are defined as

$$
\begin{aligned}
& \mathrm{PA}=\left[(Q / I)^{2}+(U / I)^{2}\right]^{1 / 2} \\
& \mathrm{PD}=\frac{1}{2} \arctan (U / Q) .
\end{aligned}
$$

The associated uncertainties on PD and PA are derived from the statistical errors on the pulsar counts measured in each of the three images. To investigate a possible dependence of the polarisation degree on the telescope roll angle chosen to align the polariser (see Sect. 2), we repeated our measurement using a different triplet of images taken with a different sequence of roll angles $\left(45^{\circ}, 90^{\circ}\right.$, and $\left.135^{\circ}\right)$ but we found no significant difference. To the statistical uncertainty of our measurement, we have to add the uncertainty on the absolute polarimetry calibration of the WFPC2, which includes the effects of the instrument intrinsic polarisation. For the $P C$ chip, this uncertainty is estimated to be of the order of $1.5 \%$ (Biretta \& Mc Master 1997). We thus assumed an overall error of $3 \%$ on the measured polarisation degree of the pulsar.

Of course, this value must be corrected for the contribution of the foreground polarisation towards PSR B0540-69, which is produced by the dust in the interstellar medium (ISM) and by the integrated components of both the Galaxy and the LMC and it is not negligible in principle. To estimate the contribution of the foreground polarisation, we measured the polarisation degree of a number of test stars uniformly distributed across the $P C$ field-of-view. We followed the same recipe as for the pulsar but through an automatic procedure for source detection and count measurement in each of the three images, aperture and CTE corrections, source list matching, and Stokes parameter computation through the WFPC2 polarisation calibration tool. For the test stars, we assumed a flat spectrum to compute the transmission of the POLQ polariser, which is accurate within $\sim 1 \%$ as a first approximation (Biretta \& Mc Master 1997). From the starting sample of test stars, we then selected those that are brighter than $m_{F 555 W}=22$ and that can thus be used as a better reference for our estimate. From these stars we then selected those $(\sim 50)$ for which the polarisation degree PD is measured with a significance of at least $3 \sigma$. After applying a $1 \sigma$ clipping filter, we ended up with a working sample of $\sim 20$ test stars from which we derived the average Stoke parameters $(Q / I)_{\text {stars }}=$ $-0.029 \%(0.023 \% \mathrm{rms})$ and $(U / I)_{\mathrm{stars}}=0.015 \%(0.029 \% \mathrm{rms})$. From these values we computed an average polarisation degree $\mathrm{PD}_{\text {stars }}=3.3 \% \pm 2.5 \%$ and an average a position angle $\mathrm{PA}_{\text {stars }} \approx 75^{\circ}$. After accounting for the $\approx 1.5 \%$ uncertainty on the 
Table 3. Compilation of the PSR B0540-69 coordinates available from the literature and associated errors $\Delta \alpha$ and $\Delta \delta$.

\begin{tabular}{llllc}
\hline \hline$\alpha_{\mathrm{J} 2000}^{(\mathrm{hms})}$ & $\Delta \alpha^{(\mathrm{s})}$ & $\delta_{\mathrm{J} 2000}^{\left(\mathrm{o}^{\prime \prime \prime}\right)}$ & $\Delta \delta^{(\prime)}$ & Source \\
\hline 054011.030 & 0.400 & -691957.50 & 2.00 & X-rays $^{1}$ \\
054011.160 & 0.150 & -691957.79 & 0.90 & Optical $^{2}$ \\
054010.980 & 0.090 & -691955.17 & 0.50 & Optical $^{3}$ \\
054011.570 & 0.080 & -691954.90 & 0.40 & X-rays $^{4}$ \\
054011.221 & 0.090 & -691954.98 & 0.50 & X-rays $^{5}$ \\
054011.173 & 0.120 & -691954.41 & 0.70 & Optical $^{6}$ \\
054011.160 & 0.040 & -691953.90 & 0.20 & X-rays $^{7}$ \\
054011.202 & 0.009 & -691954.17 & 0.05 & Optical $^{8}$ \\
\hline
\end{tabular}

Notes. ${ }^{1}$ Seward et al. (1984); ${ }^{2}$ Manchester \& Peterson (1989); ${ }^{3}$ Caraveo et al. (1992); ${ }^{4}$ Deeter et al. (1999); ${ }^{5}$ Kaaret et al. (2001); ${ }^{6}$ Serafimovich et al. (2004); ${ }^{7}$ Livingstone et al. (2005); ${ }^{8}$ this work.

absolute polarimetry calibration of the WFPC2, we then ended up with $\mathrm{PD}_{\text {stars }}=3.5 \% \pm 3 \%$, which we assumed as an estimate of the foreground polarisation. We note that our value is compatible with the $\approx 2-3 \%$ estimated by Chanan \& Helfand (1990) using a larger sample of test stars. After subtracting the foreground polarisation in the Stokes parameter space, the corrected Stokes parameters of the pulsar are then $(Q / I)_{\mathrm{PSR}}=0.12 \% \pm 0.03 \%$ and $(U / I)_{\mathrm{PSR}}=0.11 \% \pm 0.03 \%$. From these, we finally computed the intrinsic polarisation degree of the pulsar $\mathrm{PD}=16 \% \pm 4 \%$ and the polarisation position angle $\mathrm{PA}=22^{\circ} \pm 12^{\circ}$, where the attached errors also account for the rms on the Stokes parameters of the stars used to estimate the foreground polarisation.

\section{Discussion}

\subsection{The pulsar astrometry}

Unfortunately, the lack of a measurable proper motion for PSR B0540-69 does not allow searching for possible connections between the pulsar kinematics, its polarisation properties, and the PWN morphology, which have been found for the Crab and Vela pulsars (see Mignani 2009c, and references therein). The computed $3 \sigma$ upper limit of $1 \mathrm{mas} \mathrm{yr}^{-1}$ (see Sect. 3.1) on the PSR B0540-69 proper motion sets a corresponding upper limit of $\sim 250 \mathrm{~km} \mathrm{~s}^{-1}$ on its transverse velocity. Although this value is lower than the peak of the pulsar transverse velocity distribution (e.g., Hobbs et al. 2005), it does not allow claiming a peculiarly low velocity for PSR B0540-69 unless one lowers the constraints on the measured upper limit to $1 \sigma$. Indeed, several pulsars feature transverse velocities lower than $100 \mathrm{~km} \mathrm{~s}^{-1}$, e.g., including the Vela pulsar for which a velocity of $\sim 65 \mathrm{~km} \mathrm{~s}^{-1}$ has been inferred from HST astrometry (Caraveo et al. 2001).

The computed average coordinates of PSR B0540-69, together with their associated errors, are listed in Table 3, for comparison with those obtained from previous works. After its discovery by Einstein (Seward et al. 1984), the coordinates of PSR B0540-69 were first revised by Manchester \& Peterson (1989) from optical timing observations and later by Caraveo et al. (1992) from the astrometry of the optical counterpart identified in their NTT images. The discovery of the pulsar in radio (Manchester et al. 1993) did not allow more precise coordinates to be obtained. Indeed, its very low radio flux density at the LMC distance, $S_{400}=0.7 \mu \mathrm{Jy}$, makes it difficult to obtain an accurate radio timing position. Thus, the optical position of Caraveo et al. (1992) was thereafter assumed as a reference. In particular, they were used to compute the pulsar X-ray and

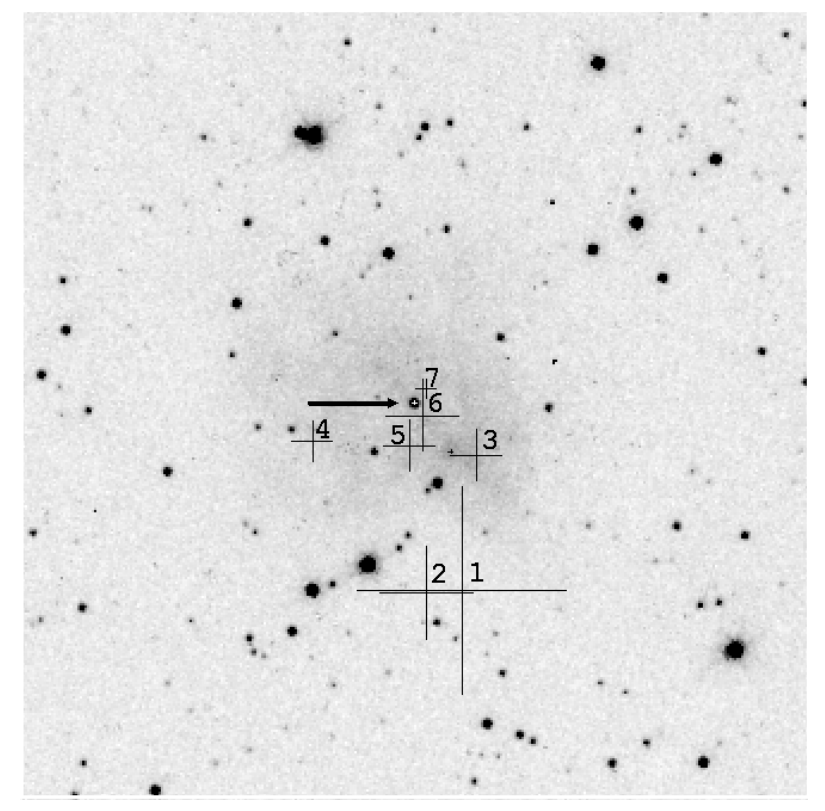

Fig. 1. 15" $\times 15^{\prime \prime}$ region of the PSR B0540-69 field observed with the HST/WFPC2 in the F555W filter (1995 October observation). North to the top, east to the left. The crosses mark the different positions of the pulsar obtained from previous X-ray and optical observations (black) and from our WFPC2 observation (white). The cross arms correspond to the $1 \sigma$ error in each coordinate. Different positions are numbered according to the reference publications (see Table 3). The pulsar is indicated by the arrow for clarity.

radio timing solution by Zhang et al. (2001), Cusumano et al. (2003), among others, but, surprisingly enough, not by Finley et al. (1993) and Manchester et al. (1993). An X-ray timing position was obtained by Deeter et al. (1999) based on Ginga observations but it was never used thereafter. More recently, Chandra observations (Kaaret et al. 2001) also yielded an updated X-ray position. The Caraveo et al. and actually not the Kaaret et al. position, as the authors claim, was still used as a reference by Johnston et al. (2004) for the pulsar X-ray/radio timing solution and by de Plaa et al. (2003). A new optical position was obtained by Serafimovich et al. (2004) from HST data, while a new X-ray timing position was derived by Livingstone et al. (2005) based on Rossi-XTE observations.

To visualise the difference and the improvement on the determination of the PSR B0540-69 position, we plotted all coordinates from Table 3 on the 1995 October WFPC2 F555W image of the field (Fig. 1). We see that the original pulsar coordinates from Seward et al. (1984) and from Manchester \& Peterson (1989) fall $\approx 3^{\prime \prime} .5$ away from the pulsar position and, actually, out of the nebula shell, while the coordinates of Deeter et al. (1999) fall right at the eastern edge of the nebula. On the other hand, the coordinates of Caraveo et al. (1992) and Kaaret et al. (2001) are indeed within the nebula but they are clearly offset from the actual pulsar position, while those of Serafimovich et al. (2004) and Livingstone et al. (2005) are more consistent with the pulsar position, although the former have much larger errors. Thus, thanks to the sharp angular resolution of the WFPC2 and to the high astrometric accuracy of $2 M A S S$, our coordinates are the most accurate obtained so far, providing a factor of $\gtrsim 4 \mathrm{im}$ provement with respect to the most recently published values. In particular, our WFPC2 coordinates supersede both the optical coordinates of Caraveo et al. (1992), which were obtained using images with lower spatial resolution (0!'13/pixel) and the 


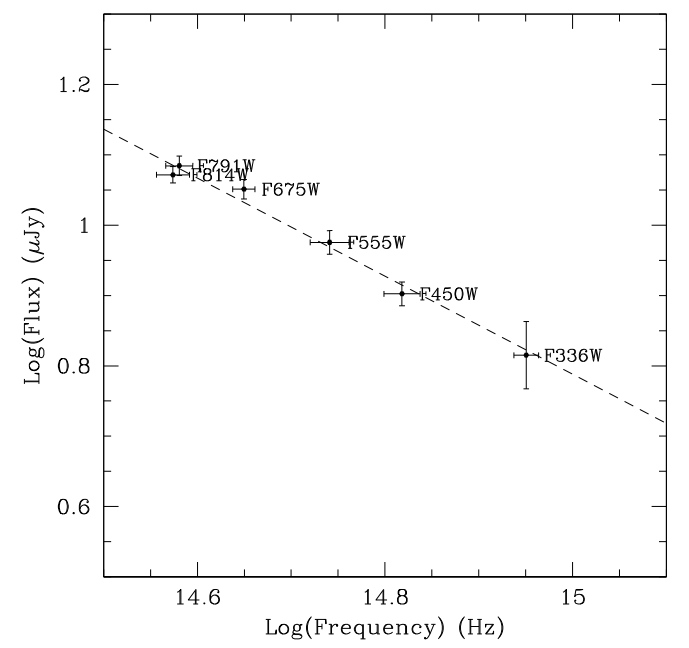

Fig. 2. Optical spectral energy distribution of PSR B0540-69 derived from the available multi-band WFPC2 photometry (Table 1). Points are labelled according to the filter names. The dashed line is to the best fit power-law spectrum.

GSC $1.0\left(\sigma_{\mathrm{s}} \sim 11^{\prime \prime}\right.$.0; Lasker et al. 1990) as a reference catalogue and those of Serafimovich et al. (2004), which were obtained using an early release of the GSC 2.0, as well as the X-ray coordinates of Kaaret et al. (2001) and Livingstone et al. (2005), obtained through Chandra imaging and Rossi-XTE timing observations, respectively.

The better determination of the PSR B0540-69 absolute position is important for follow-up non-imaging observations. In the case of spectroscopy, it will make it possible to accurately centre the pulsar in the slit when blind presets are made necessary, e.g. for ground-based observations, by the difficulties in resolving the pulsar through lower spatial resolution and seeingdominated acquisition images. As a consequence, this will allow use of narrower slits without causing any loss of signal from the pulsar. This is important for minimising the background contamination from the supernova remnant that hampered groundbased spectroscopic observations performed so far (see, e.g., Serafimovich et al. 2004).

\subsection{The pulsar spectrum}

We used our multi-band WFPC2 photometry of PSR B0540-69 to investigate its optical SED better. For consistency with previous works, we computed the interstellar extinction towards the pulsar using an $E(B-V)=0.2$ as a reference and $R=3.1$ that have been verified by several independent measurements (see Serafimovich et al. 2004, and references therein). We derived the extinction coefficients in the WFPC2 filters from the extinction curves of Fitzpatrick (1999). The pulsar SED is shown in Fig. 2, after correction for the interstellar extinction. The plotted errors on the spectral fluxes are purely statistical and do not account for the systematic 0.02-0.04 mag error on the WFPC2 zero points (Heyer et al. 2004). A linear fit to the data points yields a spectral index $\alpha_{\mathrm{O}}=0.70 \pm 0.07$. As seen, our fit clearly confirms that there is no evidence of the $U$-band excess originally claimed by Middleditch et al. (1985).

We compared our best-fit power-law spectrum with that measured by Serafimovich et al. (2004). They derived a spectral

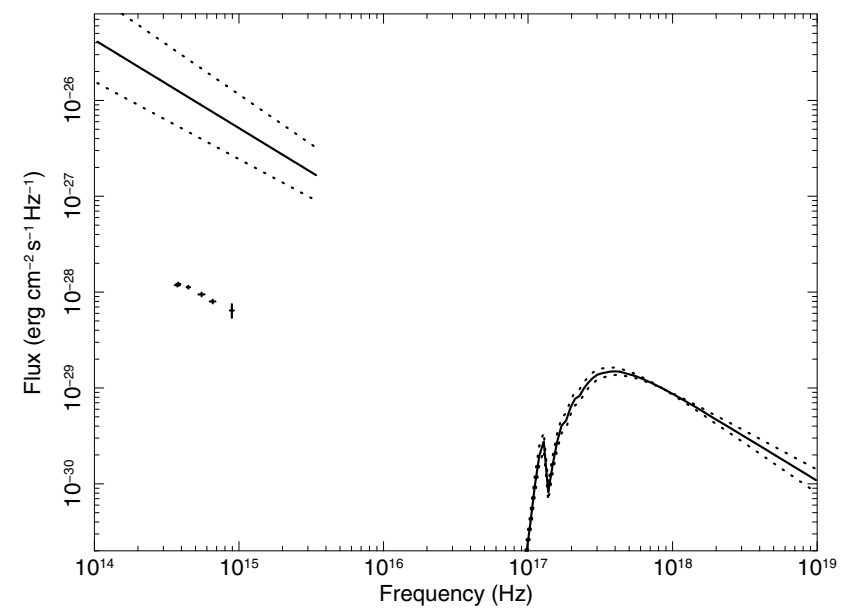

Fig. 3. Optical spectral energy distribution of PSR B0540-69 (points) compared with the power-law model (Kaaret et al. 2001) best-fitting the Chandra X-ray spectrum (solid line) and its extrapolation in the optical domain. Dashed lines correspond to a $1 \sigma$ uncertainty on the model parameters.

index $\alpha_{\mathrm{O}}=1.07_{-0.19}^{+0.20}$, which is somewhat steeper than measured by us, although not formally incompatible. As we stated above, the power-law slope is only marginally affected by the difference in the assumed interstellar extinction correction. Indeed, applying the extinction coefficients of Cardelli et al. (1989) to our spectral fluxes, as done by Serafimovich et al. (2004), yields a power law slope that is substantially identical to the one obtained using the extinction coefficients of Fitzpatrick (1999). Thus, the difference in the power-law slope is most likely intrinsic to the photometry measurements. First of all, Serafimovich et al. (2004) fitted a lower number of points (see Table 1) and used the flux measured in the medium/narrow-band F547M and F658N filters, while we fitted a larger number of points and only used the flux measured in wide-band filters. Moreover, while we used similar photometry apertures, we applied aperture corrections specific to the WFPC2, that are wavelength-dependent (Holtzman et al. 1995), and we applied the CTE correction, which apparently was not accounted for by Serafimovich et al. (2004).

The updated value of the PSR B0540-69 optical spectral index is now more consistent with those of the other optically identified pulsars, which all feature relatively steep spectral indexes $\left(\alpha_{\mathrm{O}} \gtrsim 0.4\right)$, while only the Crab and Vela pulsars feature a nearly flat power-law spectrum (see Mignani et al. 2007a, for a comparison). In particular, PSR B0540-69 may be the pulsar with the steepest optical spectral index. We also note that the new value of $\alpha_{\mathrm{O}}=0.70 \pm 0.07$ is very close to that of the X-ray spectral index $\alpha_{X}=0.83 \pm 0.13$ measured with Chandra (e.g., Kaaret et al. 2001). Interestingly, however, the optical spectrum is not the continuation of the X-ray one. Indeed, the HST spectral fluxes lie about a factor of 100 below the low-energy extrapolation of the Chandra $0.1-10 \mathrm{keV}$ X-ray spectrum (see Fig. 3). This difference cannot be accounted for by possible problems in subtracting the PWN background or by other kinds of systematic effects and confirms the presence of a spectral turnover between the optical and the X-ray bands (see also Fig. 14 of Serafimovich et al. 2004).

Spectral turnovers between these two energy bands are a common feature of the magnetospheric emission of many other rotation-powered pulsars, as indicated by the differences in their optical and X-ray spectral indexes (e.g., Mignani et al. 2007a). For comparison, we plotted in Fig. 4 the optical, near 
A\&A 515, A110 (2010)
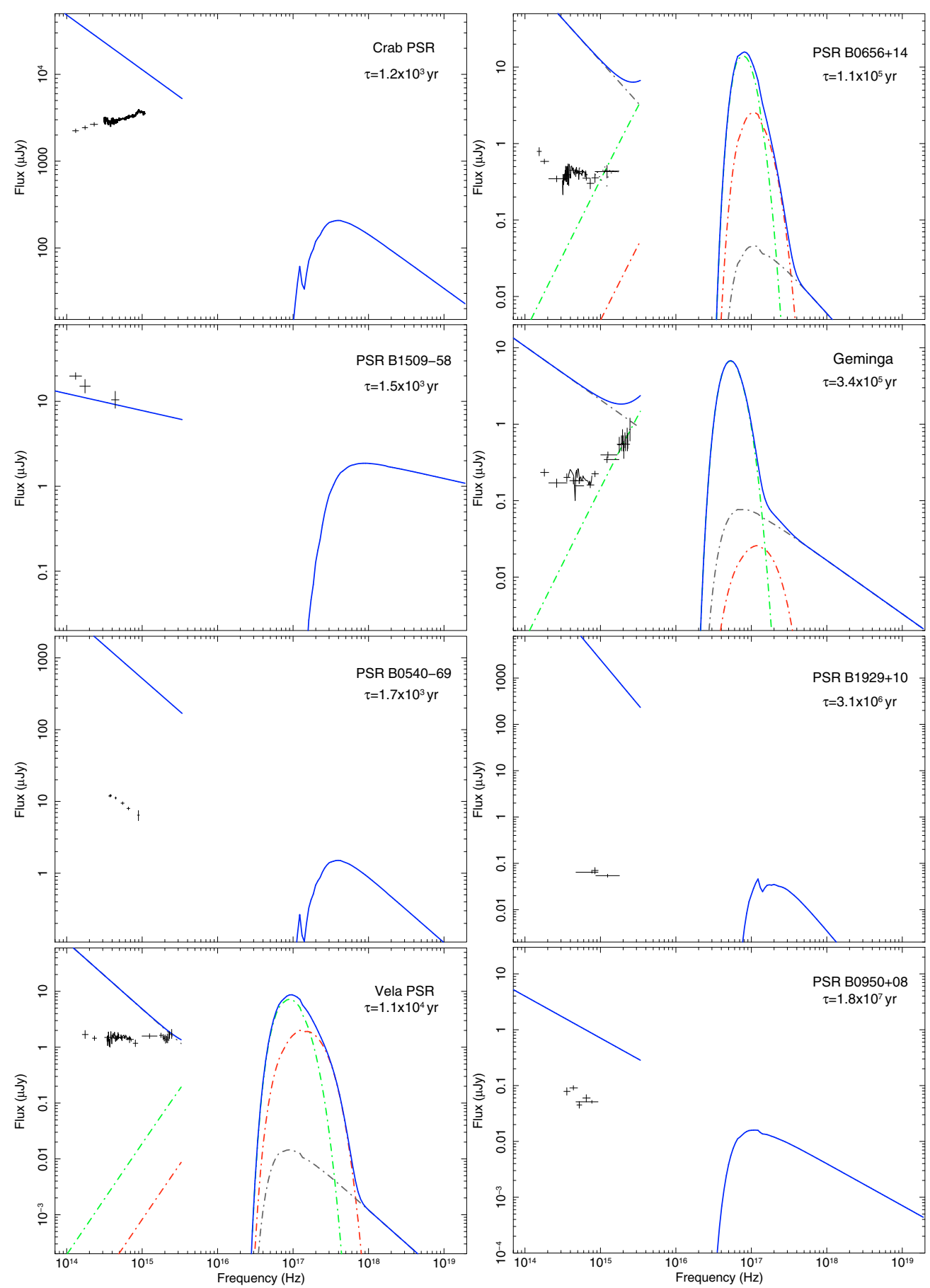

Fig. 4. Same as Fig. 3 but for all rotation-powered pulsars with an optical counterpart and flux measurements in at least two bands. Optical flux values are taken from the compilation in Mignani et al. (2007a). X-ray spectral models are taken from the source publications: Crab (Willingale et al. 2001), PSR B1509-58 (Gaensler et al. 2002), Vela (Manzali et al. 2007), PSR B0656+14 (De Luca et al. 2005), Geminga (Caraveo et al. 2004), PSR B1929+10 (Becker et al. 2006), PSR B0950+08 (Becker et al. 2004). Left panels: young pulsars $(\tau<10000$ years). Right panels: middle-aged and old pulsars ( $\tau>100000$ years). In each column, objects are sorted according to their spin-down age and labelled. Different $\mathrm{X}$-ray spectral components are shown by the dot-dashed red, green (black body) and black (power-law) lines, while the solid blue lines show the composite spectra. Only best fits are plotted for clarity.

ultraviolet (NUV), and near infrared (NIR) spectral fluxes for all rotation-powered pulsars with an optical counterpart, together with the model $0.1-10 \mathrm{keV} \mathrm{X}$-ray spectra. For the Crab and Vela pulsars, the spectral turnover is in the form of a single break in the optical-to-X-ray power-law spectrum. In the case of PSR B0540-69, instead, a double break is required to join the optical and the X-ray SEDs, unless the actual interstellar extinction is higher by a factor of 2 with respect to current best estimates, which would make the spectrum flatten in the blue. This apparent double break in the optical-to-X-ray power-law spectrum might also be present in other, much closer, rotationpowered pulsars. Of course, for some pulsars (especially for the 
fainter ones) the comparison is hampered by the paucity of spectral flux measurements at NIR/optical/NUV wavelengths, which makes it difficult to establish the presence of a power-law component. Bearing this caveat in mind, a possible double break can be recognised in the optical-to-X-ray power-law spectrum of the middle-aged pulsars PSR B0656+14 ( $\approx 100 \mathrm{kyr})$ and, to a lesser extent, Geminga ( $\approx 340 \mathrm{kyr}$ ), for both of which a powerlaw tail is hinted at in the NIR. On the other hand, a single break is probably required for the old pulsars PSR B1929+10 ( $\approx 3$ Myr) and PSR B0950+08 ( $\approx 18$ Myr), while the optical/NIR power-law seems to nicely fit the extrapolation of the X-ray one only for the young PSR B1509-58. Three different trends are therefore recognised in our sample which spans a wide range of pulsar ages. This means that the breaks in the power-law spectrum do not correlate with the neutron star age and, thus, are not indicative of any evolution in the emission processes in the neutron star magnetosphere. This is consistent with the lack of evidence for a power-law spectrum evolution, both in the optical (Mignani et al. 2007a) and in the X-rays (Becker 2009). The shape of the power-law optical-to-X-ray spectrum does not correlate with the dipole magnetic field either with, e.g. the Crab and PSR B0950+08, both featuring a single spectral break but having magnetic fields different by two orders of magnitudes. It is possible that the number of observed spectral breaks is related to the geometry of the emission regions and to the particle distribution in the neutron star magnetosphere, which are, however, difficult to reconstruct without having information on both the $\mathrm{X}$-ray and optical light curve profiles. At the same time, it is not possible to determine whether such double breaks are simply associated with a change in the power-law spectrum or they are associated with absorption processes in the neutron star magnetosphere, a possibility proposed by Serafimovich et al. (2004) for PSR B0540-69. Observations in the far UV (FUV) would be fundamental for clarifying this issue. Unfortunately, only a few pulsars have been observed in this spectral region by the EUVE satellite (Korpela \& Bowyer 1998), but only Geminga (Bignami et al. 1996) and PSR B0656+14 (Edelstein et al. 2000) have been detected amongst the pulsars shown in Fig. 4, with their FUV fluxes being more or less compatible with the extrapolations of the black body components fitting the optical spectra.

\subsection{The pulsar polarisation}

Our value for the phase-averaged polarisation ( $\mathrm{PD}=16 \% \pm 4 \%)$ is consistent with the upper limit of $15 \%$ inferred by Middleditch et al. (1987) from phase-resolved polarimetry of the pulsar. However, the measured $P D$ is higher than $\mathrm{PD} \approx 5 \%$ obtained from VLT phase-averaged polarisation observations by Wagner \& Seifert (2000), for which, however, no error estimate is given, so that its significance cannot be assessed. Thus, ours is the only significant measurement ( $\sim \sigma$ level) of the PSR B0540-69 phase-averaged polarisation obtained so far.

Measurements of the phase-averaged polarisation degree have been obtained for all the young ( $\$ 10000$ years old) rotation-powered pulsars with an optical counterpart, albeit with a different degree of confidence (see Mignani et al. 2007b; and Słowikowska et al. 2009, for a summary). For the Crab, a value of $\mathrm{PD}=9.8 \% \pm 0.1 \%$ was inferred from phaseresolved polarimetry observations (Słowikowska et al. 2009), while image polarimetry observations with the VLT yielded $\mathrm{PD}=9.4 \% \pm 4 \%$ for the Vela pulsar (Mignani et al. 2007b) and PD $=10.4 \%$ for PSR B1509-58 (Wagner \& Seifert 2000), with the latter measurement admittedly very uncertain and quoted with no error. The phase-averaged polarisation of PSR B0540-69, PD $=16 \% \pm 4 \%$, is thus consistent with the measurements obtained for the other young pulsars, as one might expect from their similar parameters, such as spin down luminosity and/or magnetic field strength. Although the current data base is still extremely limited, with the measurement obtained for PSR B1509-58 awaiting confirmation and with the phaseresolved polarimetry observations of the middle-aged ( $100 \mathrm{kyr})$ PSR B0656+14 (Kern et al. 2003) covering only one third of the period, it nonetheless suggests that the phase-averaged optical polarisation degree of rotation-powered pulsars is typically around 10\%, with our value of PD for PSR B0540-69 possibly somewhat higher.

On the theoretical side, the comparison of the observed phase-averaged polarisation degrees with the predictions of different pulsar magnetosphere models is complicated both by the degree of complexity of such models and by the limits in model simulations. However, for both the Crab and Vela pulsars, a detailed comparison with different pulsar magnetosphere models, showed that the measured phase-averaged polarisation degree of the emerging optical radiation requires the rather low degree of polarisation intrinsic to the source and/or strong depolarisation factors (Słowikowska et al. 2009; Mignani et al. 2007b). Recent 3D outer-gap model calculations of optical and/or high-energy radiation with detailed incorporation of electron-positron gyration have been carried out so far only for the Crab pulsar (Takata et al. 2007). In general, the results seem to fit the measured optical polarisation level (see Słowikowska et al. 2009) for some particular values of the viewing angle (the angle between the line of sight and the spin axis). Other models addressing the problem of polarisation characteristics are relatively more simplified in terms of the model assumptions in use (Dyks et al. 2004; Petri $\&$ Kirk 2005). All models mentioned above - relevant for young energetic pulsars - rely on spatially extended sources of emission (either within the magnetosphere - Dyks et al. 2004; Takata et al. 2007) or in the pulsar wind zone (Petri \& Kirk 2005). As a consequence, the depolarisation effects due to rotation, photon finite time of flight, and magnetic field structure are significant in all three models. This might explain the somewhat higher polarisation degree of PSR B0540-69. However, one should keep in mind that the shape of its optical light curve makes it more difficult to infer the geometry of the emission region with respect to the Crab and Vela pulsars, which provides crucial input parameters for model simulations.

Interestingly, for both the Crab and Vela pulsars, the polarisation position angle features a remarkable alignment with the axis of symmetry of the X-ray structures (torus and jets) observed by Chandra, the pulsar spin axis, and the proper motion vector (Słowikowska et al. 2009; Mignani et al. 2007b). Unfortunately, for PSR B0540-69 the scenario is not as clear since no proper motion has been measured so far, and Chandra observations (Gotthelf \& Wang 2000; Petre et al. 2007) could only partially resolve the morphology of the PWN, although its clear asymmetry might hint at the existence of a torus and, possibly, of a jet. A similar PWN morphology was also observed in the optical from HST/WFPC2 observations (Caraveo et al. 2000). Most noticeably, the WFPC2 images shows a bright emission knot southwest of the pulsar, which is apparently moving at a speed of $\sim 0.04 c$ (De Luca et al. 2007) and which can be interpreted as the head of a possible jet emitted by the pulsar. The orientation of the pulsar polarisation vector $\left(\mathrm{PA}=22^{\circ} \pm 12^{\circ}\right)$ is consistent, accounting for possible projection effects, with that of the semi-major axis of the PWN (see Fig. 5). Moreover, the pulsar phase-averaged polarisation vector is also possibly aligned with the apparent direction of motion of the knot $\left(\approx 230^{\circ}\right.$ east of 
A\&A 515, A110 (2010)

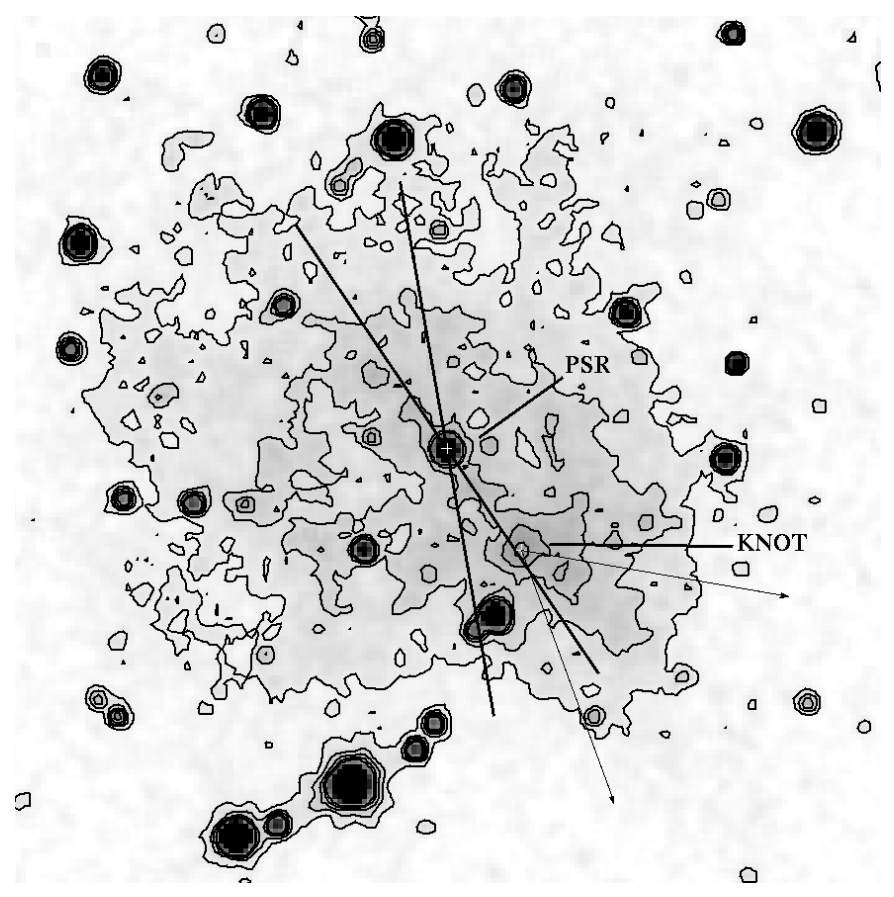

Fig. 5. Same as in Fig. 1 but zoomed over a $8^{\prime \prime} \times 8^{\prime \prime}$ region. PSR B0540-69 is labelled. The image has been smoothed over $3 \times$ 3 pixel cells for a better visualisation of the extended PWN emission. Isophotal contours (linear spacing) are drawn as solid lines. The thick lines show the measured $1 \sigma$ uncertainty $\left( \pm 12^{\circ}\right)$ on the pulsar polarisation position angle, while the thin arrows show the assumed uncertainty $\left( \pm 30^{\circ}\right)$ on the apparent proper motion direction of the bright emission knot in the PWN (De Luca et al. 2007).

north $)^{5}$. We estimated the chance alignment probability between the two vectors to be $\sim 0.04$, but still not negligible. If real, however, this peculiar alignment would indicate a possible physical connection between the pulsar and the knot, as already proposed in De Luca et al. (2007), and would support the pulsar/jet scenario. The measurement of both the knot and of the PWN polarisation structure (Mignani et al. in preparation) would reinforce this scenario further. Assuming the same PWN geometry as the Crab and Vela pulsars for PSR B0540-69, the direction of the jet would suggest that a putative torus would indeed extend along the semi-minor axis of the nebula and not along the semimajor one, as previously hypothesised from Chandra and HST observations (Gotthelf \& Wang 2000; Caraveo et al. 2000).

\section{Summary}

By using the WFPC2, we performed a comprehensive study of the LMC pulsar PSR B0540-69 in the optical domain, including astrometry, multi-band photometry, and polarimetry, down to the limits achievable with the pre-refurbished HST.

From the available multi-epoch images, we obtained the tightest possible constraints on the PSR B0540-69 proper motion $\left(<1 \mathrm{mas} \mathrm{yr}^{-1}\right)$ and transverse velocity $\left(<250 \mathrm{~km} \mathrm{~s}^{-1}\right)$. Since the WFPC2 has now been decommissioned and PSR B0540-69 has not been observed yet with $A C S$, it is unlikely that our limit will be improved by HST before its retirement around 2015 . Only the MICADO adaptive optics IR camera at the European

\footnotetext{
5 Accounting for the proper motion of the LMC (Costa et al. 2009) and for the galactic rotation does not significantly affect this apparent alignment.
}

Extremely Large Telescope $e^{6}$ (E-ELT), with an expected sensi-

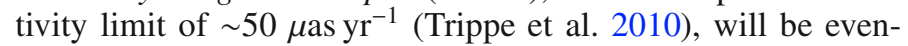
tually able to measure the pulsar proper motion. From the same WFPC2 data set, we measured the absolute position of PSR B0540-69 with an accuracy of $\sim 70$ mas, improving by a factor of 4 on the available X-ray coordinates. A further improvement could be achieved by $H S T$ using the refurbished $A C S$ or the new Wide Field Camera 3 (WFC3), which have a larger field-of-view and a smaller pixel scale with respect to the WFPC2 mosaic, and the future Gaia astrometric catalogue which will not be released in its final version, however, until 2019. From observations in six different filters, we characterised the PSR B0540-69 optical SED, as accurately as could be done through broad-band imaging photometry with the HST, confirming that the pulsar spectrum is modelled by a power-law $\left(\alpha_{\mathrm{O}}=0.70 \pm 0.07\right)$ without evidence of a previously claimed spectral turnover in the $U$ band. While multi-band photometry with $A C S$ and $W F C 3$ can certainly improve on our result, especially in the red and blue parts of the spectrum, high-spatial resolution spectroscopy with either of the two instruments or with the refurbished Space Telescope Imaging Spectrograph (STIS) is the only way to obtain a more detailed characterisation of the pulsar optical SED. Finally, from image polarimetry, we derived a new measurement of the phase-averaged polarisation degree of PSR B0540-69 (PD $=16 \% \pm 4 \%$; PA $\left.=22^{\circ} \pm 12^{\circ}\right)$, with the polarisation vector apparently aligned with the PWN semi-major axis and with the apparent proper motion direction of its bright emission knot (De Luca et al. 2007). Multi-band polarimetry observations with the refurbished, higher throughput, ACS will allow both more precise measurement of the pulsar phaseaveraged polarisation degree and of its positional angle and investigation of a possible dependence on the wavelength. Phaseresolved polarisation measurements in the optical, as well as in the $X$ and $\gamma$-rays, are crucial for testing and constraining current models of pulsar magnetosphere activity. Unfortunately, sensitive fast high-energy polarimeters, such as PoGOLite (Kamae et al. 2008), are still in the development phase, and they will not be available soon. For the time being the phase-resolved polarimetric studies in optical remain the only way to carry out such investigations. Therefore, phase-resolved optical polarimetry that, at present, can only be performed with guest instruments at ground-based telescopes, like Optima (Kanbach et al. 2009) and GASP (Collins et al. 2009), will then be unique and crucial for studying the polarisation properties of noncoherent magnetospheric radiation from PSR B0540-69 and other optical pulsars. In particular, given the faintness of PSR B0540-69 in the radio band, polarisation measurements in the optical domain are the only way to carry out such investigations.

About 25 years after its discovery, PSR B0540-69 is still the only extra galactic optical pulsar identified. Thus, it is a unique target for optical observations with the present ground and space-based facilities. More extragalactic neutron stars, however, are expected to be discovered in the optical, both in the magellanic clouds and beyond, after the advent of the E-ELT which has the potential of detecting Crab-like optical pulsars up to the distance of M 31 (Shearer 2008).

Acknowledgements. R.P.M. acknowledges STFC for support through a rolling grant and thanks G. Soutchkova (STScI) for support in the observation scheduling and S. Bagnulo and J. Dyks for useful discussions. The authors thank S. Zharikov for arranging in tabular format the data plotted in Fig. 4. A. Słowikowska is partially supported by the European Union Marie Curie grant MTKD-CT-2006-039965 and Polish Grant N N203 273833.

${ }^{6}$ http://www.eso.org/sci/facilities/eelt/ 


\section{References}

Anderson, J., \& King, I. R. 1999, PASP, 111, 1095

Anderson, J., \& King, I. R. 2003, PASP, 115, 113

Baggett, S., et al. 2002, in HST WFPC2 Data Handbook, v. 4.0, ed. B. Mobasher, Baltimore, STScI

Becker, W. 2009, in Neutron Stars and pulsars, Ap\&SS Library, 357, 91

Becker, W., Weisskopf, M. C., Tennant, A. F., et al. 2004, ApJ, 615, 908

Becker, W., Kramer, M., Jessner, A., et al. 2006, ApJ, 645, 1421

Bignami, G. F., Caraveo, P. A., Mignani, R. P., et al. 1996, ApJ, 456, L111

Biretta, J., \& Mc Master, M. 1997, Instrument Science Report WFPC2 97-11

Boyd, P. T., van Citters, G. W., Dolan, J. F., et al. 1995, ApJ, 448, 365

Campana, R., Mineo, T., De Rosa, A., et al. 2008, MNRAS, 389, 691

Caraveo, P. A., Bignami, G. F., Mereghetti, S., \& Mombelli, M. 1992, A\&A, 395,103

Caraveo, P. A., Mignani, R. P., De Luca, A., et al. 2000, Proc. of A decade of HST science, STScI, 105, 9

Caraveo, P. A., De Luca, A., Mignani, R. P., \& Bignami, G. F. 2001, ApJ, 561, 930

Caraveo, P. A., De Luca, A., Mereghetti, S., et al. 2004, Science, 305, 376

Cardelli, J. A., Clayton, G. C., \& Mathis, J. S. 1989, ApJ, 345, 245

Chanan, G. A., \& Helfand, D. J. 1990, ApJ, 352, 167

Cocke, W. J., Disney, M. J., \& Taylor, D. J. 1969, Nature, 221, 525

Collins, P. P., Shehan, B., Redfern, M., \& Shearer, A. 2009, Proc, of Polarimetry days in Rome: Crab status, theory and prospects [arXiv: 0905.0084]

Costa, E., Mendez, R. A., Pedreros, M. H., et al. 2009, AJ, 137, 4339

Cusumano, G., Massaro, E., \& Mineo, T. 2003, A\&A, 402, 647

Deeter, J. E., Nagase, F., \& Boynton, P. E. 1999, ApJ, 523, 300

De Luca, A., Caraveo, P. A., Mereghetti, S., et al. 2005, ApJ, 623, 1051

De Luca, A., Mignani, R. P., Caraveo, P. A., \& Bignami, G. F. 2007, ApJ, 667, L77

Dolphin, A. E. 2009, PASP, 121, 655

Dyks, J., Harding, H. K., \& Rudak, B. 2004, ApJ, 606, 1125

de Plaa, J., Kuiper, L., \& Hermsen, W. 2003, A\&A, 400, 1013

Edelstein, J., Seon, K.-I., Golden, A., \& Min, K.-W. 2000, ApJ, 539, 902

Finley, J. P., Ögelman, H., Hasinger, G., \& Trümper, J. 1993, ApJ, 410, 323

Fitzpatrick, E. L. 1999, PASP, 111, 63

Gaensler, B. M., Arons, J., Kaspi, V. M., et al. 2002, ApJ, 569, 878

Gotthelf, E. V., \& Wang, Q. D. 2000, ApJ, 532, L117

Gouiffes, C., Finley, J. P., \& Ögelman, H. 1992, ApJ, 394, 581

Gradari, S., Barbieri, M., Zoccarato, P., et al. 2009, MNRAS, submitted

Johnston, S., \& Romani, R. W. 2003, ApJ, 590, L95

Johnston, S., Romani, R. W., Marshall, F. E., \& Zhang, W. 2004, MNRAS, 335, 31

Heyer, I., Richardson, M., Whitmore, B., \& Lubin, L. 2004, Instrument Science Report WFPC2 2004-001

Hill, R. J., Dolan, J. F., Bless, R. C., et al. 1997, ApJ, 486, L99

Hirayama, M., Nagase, F., Endo, T., et al. 2002, MNRAS, 333, 603

Hobbs, G., Lorimer, D. R., Lyne, A. G., \& Kramer, M. 2005, MNRAS, 360, 974

Holtzman, J. A., Hester, J. J., Casertano, S., et al. 1995, PASP, 107, 156

Kaaret, P., Marshall, H. L., Aldcroft, T. L., et al. 2001, ApJ, 546, 1159

Kamae, T., Andersson, V., Arimoto, M., et al. 2008, Astrop. Phys., 30, 72
Pétri, J., \& Kirk, J. G. 2005, ApJ, 627, L37

Kanbach, G., Stefanescu, A., Duscha, S., et al. 2009, Ap\&SS Library, 351, 153 Kaplan, D. L., Chatterjee, S., Gaensler, B. M., \& Anderson, J. 2008, ApJ, 677, 1201

Kern, B., Martin, C., Mazin, B., \& Halpern, J. P. 2003, ApJ, 597, 1049

Korpela, E. J., \& Bowyer, S. 1998, AJ, 115, 2551

Lasker, B. M., Sturch, C. R., McLean, B. J., et al. 1990, AJ, 99, 2019

Lasker, B. M., Lattanzi, M. G., McLean, B. J., et al. 2008, AJ, 136, 735

Lattanzi, M. G., Capetti, A., \& Macchetto, F. D. 1997, A\&A, 318, 997

Livingstone, M. A., Kaspi, V. M., \& Gavriil, F. 2005, ApJ, 633, 1095

Manchester, R. N., \& Peterson, B. A. 1989, ApJ, 342, 23

Manchester, R. N., Mar, D. P., Lyne, A. G., et al. 1993, ApJ, 1993, 403, L29

Manzali, A., De Luca, A., \& Caraveo, P. A. 2007, ApJ, 669, 570

Middleditch, J., \& Pennypacker, C. 1985, Nature, 313, 659

Middleditch, J., Pennypacker, C. R., \& Burns, M. S. 1987, ApJ, 315, 142

Mignani, R. P. 2009a, Proc. of Neutron Stars and Gamma-ray Bursts: Recent

Developments and Future Directions, AIP, in press [arXiv:0908.1010]

Mignani, R. P. 2009b, ASpR [arXiv: 0912 . 2931]

Mignani, R. P. 2009c, ASpR, Proc, of Polarimetry days in Rome: Crab status, theory and prospects [arXiv:0902.0631]

Mignani, R. P., Pulone, L., Iannicola, G., et al. 2005, A\&A, 431, 659

Mignani, R. P., Zharikov, S., \& Caraveo, P. A. 2007a, A\&A, 473, 891

Mignani, R. P., Bagnulo, S., Dyks, J., et al. 2007b, A\&A, 467, 1157

Mineo, T., Cusumano, G., Massaro, E., et al. 1999, A\&A, 348, 519

Monet, D. G., Levine, S. E., Canzian, B., et al. 2003, AJ, 125, 984

Nagase, F., Deeter, J., Lewis, W., et al. 1990, ApJ, 351, L13

Nasuti, P., Mignani, R. P., Caraveo, P. A., \& Bignami, G. F. 1997, A\&A, 323, 839

Ögelman, H., \& Hasinger, G. 1990, ApJ, 353, L21

Pacini, F., \& Salvati, M. 1987, ApJ, 321, 447

Petre, R., Hwang, U., \& Holt, S. S. 2007, ApJ, 662, 988

Seward, F. D., Harnden, F. R. Jr., \& Helfand, D. J. 1984, ApJ, 287, L19

Serafimovich, N. I., Shibanov, Yu A., Lundqqvist, P., \& Sollerman, J. 2004, A\&A, 425, 1041

Shearer, A., Redfern, M., Pedersen, H., et al. 1994, ApJ, 423, L51

Shearer, A. 2008, in High Time Resolution Astrophysics, Ap\&SS Library, 351, 1

Skrutskie, M. F., Cutri, R. M., Stiening, et al. 2006, AJ, 131, 1163

Słowikowska, A., Kanbach, G., Borkowski, J., \& Becker, W. 2007, Proc. of the 363 WE-Heraeus Seminar on Neutron Stars and Pulsars 40 years after the discovery, MPE-Report 291, 44

Słowikowska, A., Kanbach, G., Kramer, M., \& Stefanescu, A. 2009, MNRAS, 397, 103

Takata, J., Chang, H.-K., \& Cheng, K. S. 2007, ApJ, 656, 1044

Trippe, S., Davies, R., Eisenhauer, F., et al. 2010, MNRAS, 402, 1126

Wagner, S. J., \& Seifert, W. 2000, Proc. of Pulsar Astronomy and Beyond, ASP Conf. Ser., 202, 315

Wallace, P. T., Peterson, B. A., Murdin, P. G., et al. 1977, Nature, 266, 692

Willingale, R., Aschenbach, B., Griffiths, R. G., et al. 2001, A\&A, 365, L212

Zacharias, N., Urban, S. E., Zacharias M. I., et al. 2004, AJ, 127, 3043

Zacharias, N., Finch, C., Girard, T., et al. 2009, AJ, 139, 2184

Zhang, W., Marshall, F. E., Gotthelf, E. V., et al. 2001, ApJ, 554, L17 